Mitteilungen der Österreichischen Geographischen Gesellschaft, 160. Jg., S. 430-446

(Annals of the Austrian Geographical Society, Vol. 160, pp. 430-446)

Wien (Vienna) 2018, https://doi.org/10.1553/moegg160s430

\title{
GESELLSCHAFTSNACHRICHTEN ${ }^{1)}$
}

\author{
Helmut WoHLSCHLÄGL, Wien* \\ mit 8 Tab. im Text
}

\section{INHALT}

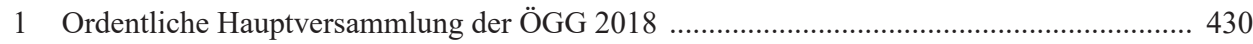

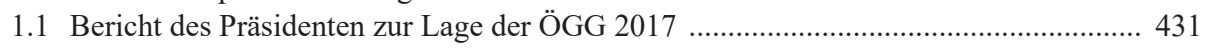

1.2 Vermögensrechnung und Rechnungsabschluss zum 31.12.2017 ................................. 435

1.3 Bericht der Rechnungsprüfer und Entlastung des Vorstandes ....................................... 438

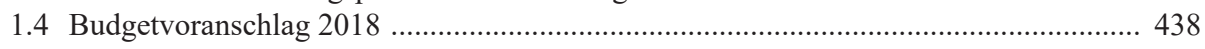

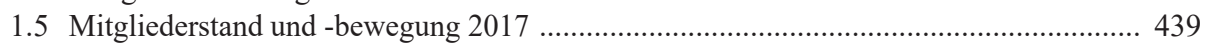

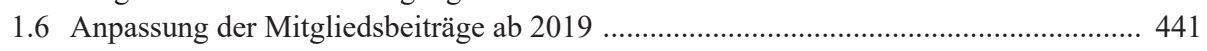

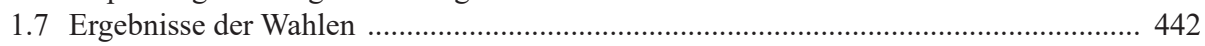

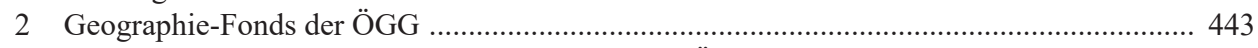

2.1 Verleihung der wissenschaftlichen Preise der ÖGG für 2016 im Dezember 2017 ........ 443

2.2 Ausschreibung der Preise des Geographie-Fonds der ÖGG für 2019 ........................... 444

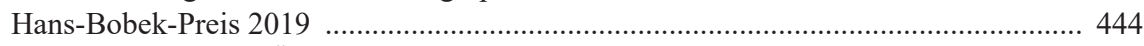

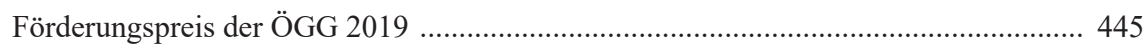

Leopold-Scheidl-Preis für Wirtschaftsgeographie 2019 ................................................ 446

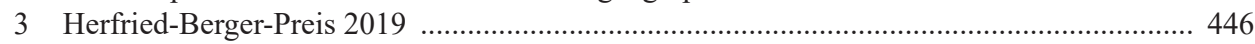

\section{Ordentliche Hauptversammlung der ÖGG 2018}

Die Einladung zur Hauptversammlung am Montag, 23. April 2018 um 18:00 Uhr im Hörsaal 5A des Instituts für Geographie und Regionalforschung, 1010 Wien, Universitätsstraße 7, 5. Stock, erfolgte in der 11. Kalenderwoche 2018 und wurde mit „Geographie aktuell“ Nr. 34 (I/2018) an alle Mitglieder zugestellt.

Die Beschlussfähigkeit der Hauptversammlung war satzungsgemäß erst ab 18:30 Uhr gegeben, da nur 18 Mitglieder anwesend waren. Gegen das in den „Mitteilungen der Österreichischen Geographischen Gesellschaft“ (MÖGG), Bd. 159/2017, S. 430-441 veröffentlichte Protokoll der Hauptversammlung 2017 vom 19. April 2017 lagen keine Einwände vor. Die für die Hauptversammlung 2018 vorgelegte Tagesordnung wurde genehmigt.

\footnotetext{
1) Zur Vereinfachung und besseren Lesbarkeit werden die akademischen Titel bei der Nennung der jeweils Zuständigen oder Berichtenden weggelassen.

* Univ.-Prof. i.R. Mag. Dr. Helmut WohlschläGL, Präsident der Österreichischen Geographischen Gesellschaft, Institut für Geographie und Regionalforschung der Universität Wien, Universitätsstraße 7/5, A-1010 Wien; E-Mail: helmut.wohlschlaegl@univie.ac.at,oegg.geographie@univie.ac.at; http://www.geoaustria.ac.at
} 


\subsection{Bericht des Präsidenten zur Lage der ÖGG 2017 (Helmut WoHLSCHLÄGL)}

Im Vereinsjahr 2017 wurde die Österreichische Geographische Gesellschaft (ÖGG) bereits 161 Jahre alt und ist damit eine der ältesten geographischen Gesellschaften der Welt sowie die drittälteste im deutschen Sprachraum nach den Geographischen Gesellschaften von Berlin (seit 1828) und Frankfurt am Main (1836) und die zweitälteste wissenschaftliche Gesellschaft in Österreich nach der Zoologisch-Botanischen Gesellschaft. Dieses kontinuierliche Bestehen einer wissenschaftlichen Gesellschaft über einen so langen Zeitraum ist nicht selbstverständlich und nicht alltäglich. Das Ziel ist über die Jahre im Wesentlichen gleich geblieben: Die Förderung und Verbreitung geographischen Wissens im Fach und über die Grenzen des Faches und der Wissenschaft hinaus. Dazu organisiert die ÖGG Vorträge, Exkursionen und weitere wissenschaftliche Veranstaltungen wie z.B. Fachausstellungen, fördert den akademischen Nachwuchs durch die Vergabe von wissenschaftlichen Preisen und gibt eine angesehene wissenschaftliche Fachzeitschrift, die „Mitteilungen der Österreichischen Geographischen Gesellschaft", heraus. Die ÖGG möchte Studierende, Wissenschaftler und alle ansprechen, die sich für geographische Themen interessieren. Sie versteht sich in diesem Sinn als Interessenvertretung der Geographinnen und Geographen an den Universitäten, in den Schulen und in den vielfältigen Arbeitsfeldern der außeruniversitären und außerschulischen Berufspraxis und ist bemüht, die Anliegen unseres Faches in der Öffentlichkeit und gegenüber anderen Institutionen zu vertreten.

- Nach den für die ÖGG wichtigen Neuerungen und Weichenstellungen im Jahr 2016, wie dem Beitritt des neu gegründeten Vereins „GeoComPass SALZBURG (Geographische Gesellschaft Salzburg)“ zur ÖGG als Mitgliedsverein und der Gründung einer neuen, vierten Fachgruppe in der ÖGG, der „Fachgruppe für Geographische und Sozioökonomische Bildung“ (GESÖB) erfolgte nun 2017 eine weitere wesentliche Neuerung, nämlich der Neustart des Exkursionsprogramms der ÖGG nach einem aktualisierten Konzept und mit einem inhaltlich ansprechenden Programm.

- Die ÖGG hat eine große Tradition in der Durchführung von Fachexkursionen. Die Chronik unserer Gesellschaft dokumentiert eine mehr als hundert Jahre lange umfangreiche und erfolgreiche Exkursionstätigkeit im In- und Ausland. Da jedoch in den letzten Jahren das Interesse der Mitglieder an Exkursionen eher gering war, fanden in den Vereinsjahren 2015 und 2016 keine Exkursionen mehr statt. und es wurde beschlossen, ein Moratorium durchzuführen, Ziele, Zielgruppen und die Gestaltung der ÖGG-Exkursionen zu überdenken und im Jahr 2017 mit einem neuen Konzept und einem interessanten Exkursionsprogramm einen Neustart durchzuführen. Ab 2017 wurde daher die Durchführung von Exkursionen wieder aufgenommen. Das neue Exkursionskonzept sieht als Kernbereich anregende Kurzexkursionen in interessante geographisch relevante Räume oder zu Institutionen vor, in die ein Zugang für Interessierte, insbesondere im Rahmen einer Spezialführung durch kompetente Fachleute, sonst nicht oder zumindest nicht so einfach möglich ist. Daneben werden mehrtägige physisch-geographisch und landschaftskundlich-kulturgeographisch ausgerichtete, ,integrative“ Exkursionen innerhalb Österreichs angeboten. Auch Auslandsexkursionen sollen wieder durchgeführt werden.

Wichtig im Rahmen des neuen Exkursionskonzepts sind auch eine laufende Auswahl interessanter Ziele, eine professionelle Bewerbung der Exkursionen, eine zeitgerechte Vorankündigung bei den Mitgliedern und eine gut organisierte Durchführung. Auch Gäste sind herzlich eingeladen, an den Exkursionen nach Maßgabe der verfügbaren freien Plätze teilzunehmen. Das neue Exkursionsprogramm bietet demgemäß aktuelle Fachinformationen und ausgewiesene 
Expertinnen und Experten, ausgewählte attraktive Ziele im In- und Ausland und interessante kultur- und physiogeographische Haltepunkte.

In diesem Zusammenhang danke ich im Namen des gesamten Vorstands sehr herzlich Herrn Jakob PACHSCHWÖLL, der als neuer Exkursionsreferent der ÖGG und als für die Exkursionen zuständiges Mitglied des Vorstandes seit dem Neustart des Exkursionsprogramms der ÖGG den Exkursionsbereich in hervorragender Weise betreut und organisiert.

- Das neue Exkursionsprogramm wurde von den Mitgliedern sofort sehr gut angenommen und im Jahr 2017 konnten bereits fünf Exkursionen angeboten werden. Besonders hinzuweisen ist auf die von Hannes HoFFERT-HösL organisierte und geleitete viertägige „Hans-Fischer-Gedenkexkursion: Österreichisches Kristallinmassiv - Alpenvorland - Nordalpen“ vom 27. bis 30. Juli 2017, an der neben ÖGG-Mitgliedern auch Studierende des Wiener Instituts für Geographie und Regionalforschung teilnahmen und die - quer über die Generationen hinweg - die von allen Teilnehmerinnen und Teilnehmern begeistert aufgenommen wurde.

Zusätzlich wurden eine Ganztagsexkursion in das Südliche Wiener Becken unter dem Titel „Entstehung, Transformation und Modernisierung eines alten Industrieraumes“ am 20. Oktober 2017, geleitet von Wolfgang Schwarz, ebenfalls mit ÖGG-Mitgliedern und Studierenden, sowie drei Kurzexkursionen angeboten, und zwar am 9. Juni 2017 in das Bezirksmuseum Josefstadt zur Sonderausstellung zum österreichischen Vermessungs- und Kartenwesen unter dem Titel „Aus der Josefstadt in die Welt. Landkarten aus dem 8ten“, am 23. Juni 2017 in das Wien Museum zur Ausstellung „Wien von oben. Die Stadt auf einen Blick“ und am 17. November 2017 in das Bundesamt für Eich- und Vermessungswesen (BEV), alle jeweils mit kompetenter Spezialführung.

Ausführlichere Berichte zu diesen Exkursionen finden sich in unserer Informationszeitschrift „Geographie aktuell“, Nr. 33 (III/2017) und Nr. 35 (I/2018).

- Weiters wurde im Jahr 2017 die Mitgliederinformation per E-Mail weiter verstärkt und auf die optisch attraktive Gestaltung sowohl mit Hilfe der bereits 2015 angeschafften Software zur Erstellung von E-Mail-Aussendungen (Mail-Designer) als auch beim Versand von Informationen mit PDF-Dateien, insbesondere bei Veranstaltungsankündigungen, Wert gelegt. In diesem Zusammenhang wurde auch die Kooperation mit der Studienvertretung Geographie und der EGEA („European Geography Association for Students and Young Geographers“) in Wien intensiviert.

- Die ÖGG nahm von 2015 bis 2017 gemeinsam mit anderen Geographischen Gesellschaften des deutschsprachigen Raums am Projekt „Neue Vermittlungsräume“ des Leibniz-Instituts für Länderkunde (IfL) in Leipzig in Kooperation mit der „Deutschen Gesellschaft für Geographie“ (DGfG) zur Entwicklung neuer zeitgemäßer Wissenstransferprodukte und Transferformate für die Vermittlung geographischen Wissens teil. Die Kooperation und Kommunikation seitens der ÖGG lag bei Robert MusiL. Im Zuge dieses Projekts fand auch ein Workshop in Wien statt und einige Zeit wurde auch ein Dissertant aus Bonn, der ein neues digitales Vermittlungsformat entwickelte, betreut. Das Projekt wurde vom Leibniz-Institut für Länderkunde Ende 2017 abgeschlossen. Prof. Sebastian Lentz zog bei der Jahresschlussveranstaltung der ÖGG ein Resümee zu diesem Projekt und präsentierte einen interessanten Abschlussbericht.

- Die farbige Informationszeitschrift für alle Mitglieder, „Geographie aktuell“, ist im Jahr 2017 mit den Heften 31, 32, 33 und 34 wie geplant regelmäßig erschienen. Die Neuerung, dass seit Anfang 2017 jedes erste und dritte Heft eines Jahrgangs mit einem um 50 Prozent erweiterten Umfang veröffentlicht wird, wodurch den Mitgliedern noch mehr aktuelle Informationen aus der 
Geographie bzw. der ÖGG, insbesondere zu Ereignissen und Aktivitäten aus den Fachgruppen, Zweigstellen und Zweigvereinen der ÖGG, aus den Geographie-Instituten, aus dem Bereich der Schulgeographie und der Berufspraxis von Geographen vermittelt werden können, wurde beibehalten. Dem Redaktionskomitee unter der Leitung von Robert MusIL und Peter A. RuMPOLT gelang es auch im Jahr 2017, dieses Medium mit zahlreichen interessanten und anregenden Beiträgen zu füllen, noch dazu nahezu kostendeckend durch Kostenbeiträge für Werbeeinschaltungen etc. Dafür ist herzlich zu danken!

- Der Jahresband der „Mitteilungen der Österreichischen Geographischen Gesellschaft“ (MÖGG) (Bd. 159/2017) ist mit 448 Seiten und 14 wissenschaftlichen Fachbeiträgen, davon zehn in englischer Sprache, mehreren kleineren Berichten und Mitteilungen, einem Abschnitt über Personalia (Würdigungen), einem umfangreichen Buchbesprechungsteil und den jährlichen Gesellschaftsnachrichten samt Rechnungsabschluss im gewohnten Umfang im Dezember 2017 erschienen und in der Jahresschlussveranstaltung der ÖGG am 5. Dezember 2017 öffentlich vorgestellt worden. Für die umsichtige und sehr umfangreiche Arbeit als Schriftleiter ist Peter JORDAN sehr herzlich zu danken!

In Ergänzung zu den bereits im Vorjahr ab dem Band 158/2016 eingeführten Neuerungen wie der Einführung eines zusätzlichen englischen Namens für unser internationales Fachjournal (,Annals of the Austrian Geographical Society“) und der zweisprachigen Angabe der Titelei, des Inhaltsverzeichnisses und aller inneren Gliederungen des Bandes im Sinne einer stärkeren Internationalisierungsstrategie der MÖGG sowie der erstmaligen „Open Access“-Verfügbarkeit aller Beiträge des Bandes auf der Homepage der ÖGG wurde nun auch für alle Fachbeiträge das DOI-System eingeführt und jedem Beitrag ein ,,digital object identifier" (doi), ein eindeutiger und dauerhafter Identifikator (DOI-Nummer), über den jeder Beitrag schnell und eindeutig im Internet zu finden ist, zugeordnet.

- Im Vereinsjahr 2017 wurden vom Stammverein Wien insgesamt sechs Vortragsveranstaltungen $^{2}$ angeboten, mit dem Ziel, den Mitgliedern der ÖGG die Möglichkeit zu bieten, im Rahmen des jeweiligen Vortrages, aber auch beim anschließenden Postkolloquium, mit den Vortragenden sowie untereinander wissenschaftlichen und geselligen Kontakt zu pflegen. Durch intensive und attraktivere Werbung und interessante Vortragsthemen gelang es, die Besucherzahl der ÖGG-Vorträge deutlich zu steigern. Die Organisation dieser Vorträge hat Wolfgang SchWARZ übernommen, dem für diesen Einsatz herzlich zu danken ist!

- Zahlreiche in der Kanzlei der ÖGG eingelangte Anfragen aus Österreich, Deutschland und anderen europäischen Staaten, die das Archiv der ÖGG oder älteres Kartenmaterial aus unserer Bibliothek, schwerpunkthaft aus der Zeit der „k.k. Geographischen Gesellschaft in Wien“ zwischen 1856 und 1914 betreffen, konnten dankenswerterweise von unserem für Ehrungen, Traditionspflege und das Archiv zuständigen Vorstandsmitglied Gerhard FASCHING erfolgreich und zur Zufriedenheit der Nachfrager bearbeitet werden.

- Der Präsident und mehrere Vorstandsmitglieder nahmen im Februar 2017 am Begräbnis des langjährigen Ehrenmitglieds der ÖGG, Frau Emer. O. Univ.-Prof. Dr. Dr. h.c. Elisabeth LıchTENBERGER teil. Ein Kranz der ÖGG wurde von Gerhard FASCHING überbracht.

\footnotetext{
2) Zu den einzelnen Vortragstiteln in Wien sowie zu den Vorträgen im Zweigverein Innsbruck, in den Zweigstellen Graz und Klagenfurt sowie beim Mitgliedsverein in Salzburg siehe „Geographie aktuell“ Nr. 31, 32, 33 und 34 .
} 
- Die Jahresschlussveranstaltung (Weihnachtsfeier) der ÖGG fand am 5. Dezember 2017 in feierlichem Rahmen im Hörsaal 5A des Instituts für Geographie und Regionalforschung der Universität Wien statt. Bei der Veranstaltung wurden im Anschluss an eine Kurzvorstellung der Preisträger/innen und eine Kurzpräsentation der preisgekrönten Arbeiten die wissenschaftlichen Preise der ÖGG durch den Präsidenten, Helmut WoHLSChLäGL, und den Geschäftsführer des Geographie-Fonds der ÖGG, Albert HOFMAYER, überreicht, weiters der neue Band der MÖGG kurz vorgestellt und schließlich - nach einer herzlichen Würdigung der Geehrten durch Albert HOFMAYER - die feierliche Verleihung der Ehrenmitgliedschaft an die langjährigen und verdienstvollen Vorstandsmitglieder unserer Gesellschaft, Brigadier i.R. Prof. Dr. Gerhard FASCHING und Hofrat i.R. Univ.-Doz. Dr. Wolfgang SchwaRz, durchgeführt. Eine Würdigung der zwei neuen Ehrenmitglieder mit näheren Informationen findet sich in „Geographie aktuell“", Nr. $35(\mathrm{I} / 2018)$ auf Seite 7.

Unbestreitbarer Höhepunkt der Jahresschlussveranstaltung war aber der ausgezeichnete Gastvortrag von Univ.-Prof. Dr. Sebastian LenTz (Universität Leipzig, Direktor des Leibniz-Instituts für Länderkunde, IfL) zum Thema „,Geographische Gesellschaften und Wissenstransfer: Herausforderungen und Potenziale“. Dieser Vortrag war in mehrfacher Hinsicht bemerkenswert und anregend: Zum einen bot er einen interessanten Abschlussbericht zu dem Projekt „Neue Vermittlungsräume“ des IfL, an dem sich auch die ÖGG beteiligt hat, zur anderen thematisierte er ausführlich die Rolle und Funktion Geographischer Gesellschaften in der heutigen Zeit.

Anschließend gab es beim weihnachtlichen Buffet die Gelegenheit, sich auszutauschen und die Preisträger, den Gastvortragenden und die neuen Ehrenmitglieder näher kennenzulernen. Ein Betrag von Prof. Lentz über „Geographische Gesellschaften und Wissenstransfer“, in dem er wichtige Ausführungen seines Vortrags noch einmal kurz zusammengefast hat, findet sich in „Geographie aktuell“, Nr. 35 (I/2018) auf Seite 13.

- Die Zweigvereine, Zweigstellen und Fachgruppen, also jene Teilorganisationen, welche entweder regional oder fachlich näher an den Mitgliedern sind, laufen überall dort, wo Engagement und freiwilliger Einsatz vorhanden sind, sehr gut und sind so Hoffnungsträger und Aktivposten der ÖGG. Es sei daher den Leitern und Leitungsgruppen in Innsbruck, Graz und Klagenfurt a.W. sowie jenen der vier ÖGG-Fachgruppen „Österreichische Kartographische Kommission“ (ÖKK), „Österreichische Forschungsgruppe für Geomorphologie und Umweltwandel“ (geomorph.at), „Österreichischer Verband für Angewandte Geographie“ (ÖVAG) und „Fachgruppe für Geographische und Sozioökonomische Bildung“" (GESÖB) herzlichst gedankt!

Die Fachgruppen berichten laufend in unregelmäßigen Abständen über ihre Aktivitäten in unserer vierteljährlichen Informationszeitschrift „Geographie aktuell“. Hinzuweisen ist in diesem Zusammenhang für das Jahr 2017 auf den Bericht der „Fachgruppe für Geographische und Sozioökonomische Bildung“ (GESÖB) in Heft 31 (I/2017), auf den Bericht der Fachgruppe „Österreichische Kartographische Kommission“ (ÖKK), ebenfalls in Heft 31 (I/2017), sowie auf den Bericht der Fachgruppe „Österreichischer Verband für Angewandte Geographie“ (ÖVAG) in Heft 33 (III/2017).

Von den Veranstaltungen und Initiativen der Fachgruppen sind in diesem Zusammenhang besonders zu erwähnen: Die Teilnahme der Fachgruppe „geomorph.at" an der gemeinsamen schweizerisch-österreichischen Jahrestagung 2017 der „Schweizerischen Geomorphologischen Gesellschaft" in Zermatt sowie das Jahrestreffen der Fachgruppe „geomorph.at“ im September 2017 in Johnsbach, weiters der jedes Jahr im Herbst in Wien von der Fachgruppe ÖVAG veranstaltete „Tag der Angewandten Geographie“ (diesmal am 24. November 2017) sowie die ebenfalls vom ÖVAG durchgeführte, ausgezeichnet besuchte Veranstaltung „GEO Talk“ am 7. 
Juni 2017 mit dem Planungsdirektor des New Yorker Stadtteils Brooklyn und die Organisation und Durchführung des für den Schulbereich und die Fachdidaktik wichtigen Symposiums „Wirtschaftliche Allgemeinbildung hat Zukunft: 55 Jahre Geographie und Wirtschaftskunde“ durch die Fachgruppe GESÖB gemeinsam mit der Arbeitsgruppe Fachdidaktik Geographie und wirtschaftliche Bildung des Instituts für Geographie und Regionalforschung an der Universität Wien in der Oesterreichischen Nationalbank in Anwesenheit der damaligen Bundesministerin für Bildung und des Gouverneurs der Nationalbank.

Das alles ist ohne die freiwillige und ehrenamtliche Mitarbeit, insbesondere aller Vorstandsmitglieder, und ohne die finanziellen Beiträge der Mitglieder nicht möglich! Ihnen allen gilt deshalb abschließend mein besondere Dank!

\subsection{Vermögensrechnung und Rechnungsabschluss zum 31.12.2017 \\ (Norbert HaCKNER-JAKLIN, Helmut WOHLSCHLÄGL)}

Aus der Sicht des Rechnungswesens konnte das Wirtschaftsjahr 2017, wie aus den detaillierten Aufstellungen in Tabelle 1 und 2 hervorgeht, ordnungsgemäß abgewickelt werden und die ÖGG ihre Aufgaben voll wahrnehmen. Sämtliche Einnahmen und Ausgaben haben sich im Wesentlichen - mit geringen Ausnahmen - budgetgemäß entwickelt. Nachfolgend einige Erläuterungen zum Zahlenwerk:

- Das Rechnungsjahr 2017 wurde praktisch ausgeglichen, mit nur einem kleinen operativen Verlust von EUR 136,97 abgeschlossen.

- Die Einnahmen aus Mitgliedsbeiträgen waren leicht rückläufig, vor allem, weil im Vorjahr durch eine Aktion zur Verstärkung des Mahn- und Erinnerungswesens für ausstehende Mitgliedsbeiträge eine Reihe größerer Nachzahlungen erfolgte, was sich 2016 in einem leicht erhöhten Aufkommen an Mitgliedsbeitragen niederschlug. Dieser „Nachzahlungseffekt“ war 2017 nicht mehr in diesem Ausmaß gegeben. Allerdings sind im Jahr 2017 die Spenden im Vergleich zum Vorjahr deutlich angestiegen, bewegen sich aber in Summe noch immer auf einem niedrigen Niveau.

- Die Einnahmen aus Publikationsverkäufen haben sich erfreulicherweise im Vergleich zum Vorjahr mehr als verdoppelt.

- Die Höhe des (geringen) Verlusts konnte demgemäß gegenüber dem Vorjahr deutlich reduziert werden. Die Abweichungen gegenüber dem Budget sind einnahmenseitig vor allem auf die gestiegenen Erlöse aus dem Publikationsverkauf und die erstmalige Einwerbung eines Förderungsbetrages in der Höhe von EUR 1.000,- als Druckkostenzuschuss für den Band 159 (2017) der MÖGG bei der Kulturabteilung der Stadt Wien, Abteilung Wissenschafts- und Forschungsförderung, und ausgabenseitig auf die gestiegenen Verwaltungskosten und die deutlich höheren Vortragskosten zurückzuführen.

- Andererseits ist zu vermerken, dass es gelang, die Herstellungskosten der MÖGG für den Band 158/2016 durch die Einstellung der Produktion von Sonderdrucken für die Autorinnen und Autoren neuerlich um rund EUR 1.000,- zu senken. Diese Kostenabsenkung wurde im Jahr 2017 budgetwirksam, da der Band etwas verspätet erst im Jänner 2017 erschienen ist. 
- Der Geographie-Fonds konnte seine Aufgaben voll wahrnehmen und eine ordnungsgemäße Preisgeldverleihung durchführen. Primär bedingt durch Bewertungsgewinne in der Höhe von EUR 2.886,50 konnte der Fonds ein positives Gesamtergebnis von EUR 872,62 erwirtschaften. Aufgrund der derzeit nach wie vor bestehenden niedrigen Zinsertragslage ist das operative Ergebnis des Geographie-Fonds trotz Spendeneinnahmen mit EUR 2.013,88 leicht negativ, wird aber durch die bereits erwähnten Bewertungsgewinne deutlich aufgewogen.

\begin{tabular}{|c|c|c|c|}
\hline \multicolumn{2}{|l|}{ AKTIVA } & \multicolumn{2}{|l|}{ PASSIVA } \\
\hline Anlagevermögen & & Kapital & \\
\hline Inventar Geschäftsstelle(Wandsystem) & 0,00 & Kapital & $1.409,98$ \\
\hline Bibliothek & 0,00 & & \\
\hline Kaution Kanzlei & 700,00 & Rücklagen & \\
\hline & & Allgemeine Rücklage & $11.512,65$ \\
\hline Umlaufvermögen & & Rücklage Investitionsfonds & $13.992,24$ \\
\hline Forderungen (offene Rechnungen) & $1.157,30$ & Rücklage Fachgruppe ÖKK & $7.740,87$ \\
\hline Ausstehende Mitgliedsbeiträge & $5.641,00$ & Rücklage Fachgruppe geomorph.at & $10.595,56$ \\
\hline & & Rücklage Fachgruppe ÖVAG & $1.043,67$ \\
\hline Bankguthaben & & Rücklage Exkursion Inland & 863,67 \\
\hline Giro Erste Bank ÖGG & $1.767,28$ & Rücklage Exkursion Ausland & $1.354,45$ \\
\hline Subkonto Fachgruppe ÖKK & $1.166,81$ & Rücklage Geographie-Fonds & $156.097,16$ \\
\hline Subkonto Fachgruppe geomorph.at & $6.952,88$ & & \\
\hline Subkonto Fachgruppe ÖVAG & $1.451,63$ & Rückstellungen & \\
\hline Profitkonto Erste Bank ÖGG & $32.092,35$ & Druckkostenrückstellung & $34.820,38$ \\
\hline Sparbuch Deniz Bank ÖGG & $34.093,55$ & Sonderrückstellung MÖGG & 0,00 \\
\hline Barvermögen & & Bobek-Preis & 0,00 \\
\hline Kassa Geschäftsstelle Wien & 0,00 & Förderungspreis & 0,00 \\
\hline Kassa Zweigstelle Graz & 423,52 & & \\
\hline Kassa Zweigstelle Klagenfurt a.W. & 663,31 & & \\
\hline Kassa Fachgruppe ÖKK & 0,14 & & \\
\hline Geographie-Fonds & & & \\
\hline Pfandbriefe ÖGW & $3.544,00$ & & \\
\hline Wertpapiere Depot Bank Austria & $42.233,50$ & & \\
\hline Referenzkonto Bank Austria & 390,20 & & \\
\hline Sparkonto Vakif-Bank & $51.218,75$ & & \\
\hline Profitkonto Erste Bank Geographie-Fonds & $48.336,66$ & & \\
\hline Subkonto Erste Bank Geographie-Fonds & 226,94 & & \\
\hline Bausparvertrag & $7.370,31$ & & \\
\hline & $239.430,63$ & & $239.430,63$ \\
\hline
\end{tabular}

Tab. 1: Vermögensrechnung der ÖGG per 31.12.2017 in Euro 


\begin{tabular}{|c|c|c|c|}
\hline \multicolumn{2}{|l|}{ AUFWENDUNGEN } & \multicolumn{2}{|l|}{ ERLÖSE } \\
\hline Publikationen & & Publikationen & \\
\hline Herstellung MÖGG & $10.117,10$ & Verkauf Publikationen & $3.227,20$ \\
\hline Versandkosten MÖGG (ohne Porto) & 0,00 & & \\
\hline „Geographie aktuell“" & $2.888,42$ & „Geographie aktuell“" & $3.300,00$ \\
\hline Abschr. uneinbringlicher Forderungen & 42,70 & & \\
\hline Fachgruppen & & Fachgruppen & \\
\hline ÖKK & $2.530,70$ & ÖKK & $2.515,10$ \\
\hline geomorph.at & $4.930,17$ & geomorph.at & $3.926,72$ \\
\hline ÖVAG & 48,46 & ÖVAG & 300,11 \\
\hline Exkursionen & & Exkursionen & \\
\hline Exkursion Inland & $3.917,85$ & Exkursion Inland & $4.508,80$ \\
\hline Exkursion Ausland & 0,00 & Exkursion Ausland & 0,00 \\
\hline Büroaufwand & & Mitgliedsbeiträge und Spenden & \\
\hline Raumaufwand & & Mitgliedsbeiträge & $22.662,32$ \\
\hline Miete (inkl. Instandhaltung) $3.011,05$ & & Spenden & 738,00 \\
\hline Raumpflege $\quad 0,00$ & & & \\
\hline Strom, Gas & & Subventionen & \\
\hline Versicherungen & $3.478,91$ & Subvention MÖGG & $1.000,00$ \\
\hline Kanzlei, Verwaltung & $6.138,27$ & & \\
\hline Büroaufwand & 69,36 & & \\
\hline Portogebühren & $3.620,77$ & & \\
\hline Telefongebühren, Internet & 373,20 & & \\
\hline AfA-Anlagen (Wandsystem) & 0,00 & & \\
\hline Sonstiger Druckaufwand & 39,60 & & \\
\hline Sonstige Vereinstätigkeiten & & Sonstige Vereinstätigkeiten & \\
\hline Beiträge zu Vereinen & 100,00 & & \\
\hline Bücher, Zeitschriften & 324,80 & & \\
\hline Modernisierung Bibliothek & 0,00 & & \\
\hline Vortragskosten & $3.091,00$ & & \\
\hline Reisekosten & 0,00 & & \\
\hline Repräsentation, Ehrungen & 850,77 & & \\
\hline Geographie-Fonds & & Geographie-Fonds & \\
\hline Aufwendungen Geographie-Fonds & $3.372,80$ & Erlöse Geographie-Fonds & $4.245,42$ \\
\hline Sonstiger Aufwand & & Sonstige Erlöse & \\
\hline Bankspesen & 409,96 & Kapitalerlöse & 373,17 \\
\hline Sonstiger Aufwand & 483,75 & Sonstige Erlöse & 0,00 \\
\hline Dotierung Rückstellungen & & Auflösung Rückstellungen & \\
\hline Sonderrückstellung MÖGG & 0,00 & Sonderrückstellung MÖGG & 945,00 \\
\hline Rückstellung Inlandsexkursion & 0,00 & Rückstellung Inlandsexkursion & 0,00 \\
\hline Dotierung Rücklagen & & Auflösung Rücklagen & \\
\hline Allgemeine Rücklage & 0,00 & Allgemeine Rücklage & 136,97 \\
\hline Rücklage ÖKK & 0,00 & ÖKK & 15,60 \\
\hline Rücklage geomorph.at & 0,00 & geomorph.at & $1.003,45$ \\
\hline Rücklage ÖVAG & 251,65 & ÖVAG & 0,00 \\
\hline Rücklage Investitionsfonds & 0,00 & Investitionsfonds & 0,00 \\
\hline Rücklage Geographie-Fonds & 872,62 & Geographie-Fonds & 0,00 \\
\hline & $47.952,86$ & & $47.952,86$ \\
\hline
\end{tabular}

Tab. 2: Rechnungsabschluss mit Jahresabgrenzungen per 31.12.2017 in Euro 


\subsection{Bericht der Rechnungsprüfer und Entlastung des Vorstandes}

(Andreas Palkovics und Klaus Wilhelmer)

„Die Prüfung des Rechnungsabschlusses für das Geschäftsjahr 2017 fand am 10.04.2018 in Anwesenheit des Rechnungsführers, Mag. Norbert Hackner-JAKLIN, des Präsidenten Univ.-Prof. Mag. Dr. Helmut Wohlschlägl und des 1. Vizepräsidenten, Univ.-Doz. Dr. Christian Staudacher, in den Räumlichkeiten der ÖGG durch die beiden Rechnungsprüfer, Mag. Andreas PaLKovics und Dr. Klaus WiLHELMER, statt.

Die Erstellung der Bilanz und der Gewinn/Verlust-Rechnung erfolgten zeitgerecht und konnten den Rechnungsprüfern bereits eine Woche vor dem Prüfungstermin zugestellt werden.

Im Rahmen der Rechnungsprüfung wurden sämtliche Unterlagen offengelegt und sowohl stichprobenartig Aufwands- und Erlöspositionen der Gewinn/Verlust-Rechnung geprüft als auch Bilanzpositionen erörtert. Alle Fragen wurden beantwortet und konnten inhaltlich geklärt werden. Die Überprüfung der Gebarung durch die Rechnungsprüfer erstreckte sich auf ziffernmäßige Richtigkeit, Rechtmäßigkeit, Sparsamkeit, Wirtschaftlichkeit und Zweckmäßigkeit.

1. Zusammenfassend wird wie folgt festgehalten:

a) Sämtliche Prüfungsunterlagen (Bilanz und Gewinn/Verlust-Rechnung) wurden vorgelegt.

b) Die Ausführung und die Rechnungsführung entsprechen vollinhaltlich den Grundsätzen ordnungsgemäßer Buchführung.

c) Das pagatorische Rechnungswesen wurde formell richtig geführt, insbesondere wurde der Rechnungsabschluss zum 31.12.2017 ordnungsgemäß abgeleitet. Das ausgewiesene Vermögen ist laut Bestand vorhanden und die Ausgaben sind durch Belege nachgewiesen.

2. Antrag auf Entlastung des Vorstandes: Es wird festgehalten, dass die Mittel des Vereins statutengemäß verwendet worden sind. Aus diesem Grund stellen die unterzeichneten Rechnungsprüfer den Antrag, den Rechnungsabschluss 2017 zu genehmigen und den Rechnungsführer und den Vorstand zu entlasten.“

Der Antrag auf Entlastung des Vorstandes wurde in der Hauptversammlung vom 23. April 2018 einstimmig angenommen.

\subsection{Budgetvoranschlag 2018}

(Norbert HaCKNER-JAKLIN, Helmut WoHLSCHLÄGL)

Der Budgetvoranschlag wurde wie üblich nach den Erfahrungen des abgelaufenen Vereinsjahres sowie den sich abzeichnenden Notwendigkeiten des laufenden Jahres konzipiert. Unter diesen Rahmenbedingungen wurde ein ausgeglichenes Budget für 2018 erstellt.

Auf der Ausgabenseite wurden vor allem die Portokosten, die Vortragskosten und die Aufwendungen für Bücher und Zeitschriften reduziert.

Auf der Einnahmenseite wird zwar eine Erhöhung der Einnahmen aus Mitgliedsbeiträgen und Spenden sowie der Werbeeinnahmen durch Inserate in „Geographie aktuell“ angestrebt, dies wurde aber im Budgetvoranschlag vorsichtshalber nicht berücksichtigt. Erwartet wird aber eine Reduktion der im Vorjahr überdurchschnittlich hohen Einnahmen aus dem Publikationsverkauf. 


\begin{tabular}{|c|c|c|c|c|c|c|c|}
\hline \multirow{2}{*}{$\begin{array}{l}\text { Ausgaben } \\
\text { (in } 1.000 \text { EUR) }\end{array}$} & \multicolumn{2}{|c|}{2017} & \multirow{2}{*}{\begin{tabular}{c|}
2018 \\
Soll
\end{tabular}} & \multirow{2}{*}{$\begin{array}{l}\text { Einnahmen } \\
\text { (in } 1.000 \text { EUR) }\end{array}$} & \multicolumn{2}{|c|}{2017} & \multirow{2}{*}{$\begin{array}{r}2018 \\
\text { Soll }\end{array}$} \\
\hline & Soll & Ist & & & Soll & Ist & \\
\hline Publikationen & & & & Publikationen & & & \\
\hline Herstellung MÖGG & 10,68 & 10,12 & 10,70 & Publikationsverkauf & 1,30 & 3,22 & 2,20 \\
\hline Versand MÖGG & 0,00 & 0,00 & 0,00 & & & & \\
\hline Sonstige Druckwerke & 0,00 & 0,00 & 0,00 & & & & \\
\hline „Geographie aktuell““ & 2,60 & 2,89 & 2,90 & „Geographie aktuell““ & 3,50 & 3,30 & 3,30 \\
\hline Büroaufwand & & & & Mitgliedsbeiträge & & & \\
\hline Raumaufwand & 3,50 & 3,48 & 3,50 & und Spenden & & & \\
\hline Kanzlei, Verwaltung & 6,00 & 6,14 & 6,30 & Mitgliedsbeiträge & 24,00 & 22,67 & 22,85 \\
\hline Büroaufwand & 0,10 & 0,10 & 0,10 & Spenden & 0,20 & 0,74 & 0,70 \\
\hline Portogebühren & 3,20 & 3,62 & 2,60 & & & & \\
\hline Telefongebühren, Internet & 0,45 & 0,37 & 0,40 & Sonstige Erlöse & & & \\
\hline & & & & Sonstige Erlöse & 0,30 & 0,00 & 0,00 \\
\hline Sonstige Vereinstätigkeiten & & & & Kapitalerlöse & 0,50 & 0,37 & 0,30 \\
\hline Beiträge zu Vereinen & 0,02 & 0,10 & 0,10 & Subventionen & & 1,00 & 1,00 \\
\hline Bücher, Zeitschriften & 0,30 & 0,32 & 0,00 & Überschuss Exkursionen & & 0,59 & 0,00 \\
\hline Bibliothek & 0,00 & 0,00 & 0,00 & & & & \\
\hline Vortragskosten & 1,45 & 3,09 & 2,00 & & & & \\
\hline Reisekosten & 0,15 & 0,00 & 0,00 & & & & \\
\hline Veranstaltungen, Ehrungen & 0,70 & 0,85 & 0,80 & & & & \\
\hline Sonstiger Aufwand & & & & & & & \\
\hline Bankspesen & 0,35 & 0,41 & 0,45 & & & & \\
\hline Sonstiger Aufwand & 0,30 & 0,48 & 0,50 & & & & \\
\hline AfA & 0,00 & 0,00 & 0,00 & & & & \\
\hline Zweigstellen & 0,00 & 0,00 & 0,00 & & & & \\
\hline & 29,80 & 31,97 & $\mathbf{3 0 , 3 5}$ & & 29,80 & 31,89 & 30,35 \\
\hline
\end{tabular}

Tab. 3: Budgetvoranschlag 2018 in 1.000 EUR (ohne den Zweigverein Innsbruck)

\subsection{Mitgliederstand und -bewegung 2017}

(Helmut WoHLSCHLÄGL)

GEDENKEN für die im Jahr 2017 verstorbenen Mitglieder: Im Vereinsjahr haben uns folgende Mitglieder für immer verlassen:

Dipl.-Ing. Dr. Mirjanka Lechthaler, Wien

Emer. O. Univ.-Prof. Dr. Dr.h.c. Elisabeth LichtenBerger, Wien

Hofrat Mag. Dr. Bernhard Ludwig, Wien

OStR Prof. Wolfmar STIEGLER, Wien

OStR Prof. Dr. Hertha WaLdmann, Wien

Die ÖGG wird den verstorbenen Mitgliedern ein ehrendes Andenken bewahren.

Die Mitgliederentwicklung der ÖGG zeigt insgesamt im Jahr 2017 zwar eine bessere Bilanz als in den Vorjahren, ist aber nach wie vor nicht zufriedenstellend. Die Zahl der Austritte und Strei- 
chungen sowie der Todesfälle überwog knapp jene der Eintritte, so dass sich - trotz der in den letzten Jahren kontinuierlich zunehmenden Zahl der Eintritte - auch 2017 neuerlich eine schwach rückläufige Entwicklung der Mitgliederzahl ergab. Ab 2018 soll daher verstärktes Augenmerk auf die Mitgliederwerbung, insbesondere beim jüngeren wissenschaftlichen Nachwuchs, bei den Studierenden und bei den Lehrkräften an den Schulen gelegt werden. Eine wichtige Rolle werden in diesem Zusammenhang die Beitritte zur neu gegründeten Fachgruppe GESÖB spielen.

Gleichzeitig wird es weiterhin erforderlich sein, einige Mitglieder, von denen der Mitgliedsbeitrag schon seit vielen Jahren ausständig ist und mit denen es auch nicht möglich war, wegen einer Änderung der Wohnadresse, die der ÖGG nicht mitgeteilt wurde, einen persönlichen oder schriftlichen Kontakt herzustellen, zu streichen.

\begin{tabular}{|c|c|c|c|c|c|c|c|c|c|}
\hline & \multicolumn{3}{|c|}{$\begin{array}{c}\text { Stammverein } \\
\text { Wien }\end{array}$} & \multicolumn{3}{|c|}{$\begin{array}{c}\text { Zweigverein } \\
\text { Innsbruck }\end{array}$} & \multicolumn{3}{|c|}{$\begin{array}{c}\text { ÖGG } \\
\text { gesamt }\end{array}$} \\
\hline & $\mathbf{m M}$ & $\mathbf{o M}$ & $\Sigma$ & $\mathbf{m M}$ & $\mathbf{o M}$ & $\Sigma$ & mM & $\mathbf{o M}$ & $\Sigma$ \\
\hline Ehrenmitglieder gesamt & 23 & 0 & 23 & 2 & 0 & 2 & 25 & 0 & 25 \\
\hline persönliche Ehrenmitglieder in Österreich & 12 & 0 & 12 & 2 & 0 & 2 & 14 & 0 & 14 \\
\hline persönliche Ehrenmitglieder im Ausland & 3 & 0 & 3 & 0 & 0 & 0 & 3 & 0 & 3 \\
\hline institutionelle Ehrenmitglieder Ausland & 8 & 0 & 8 & 0 & 0 & 0 & 8 & 0 & 8 \\
\hline Lebenslange Mitglieder & 4 & 0 & 4 & 0 & 0 & 0 & 4 & 0 & 4 \\
\hline $\begin{array}{l}\text { Ordentliche Mitglieder gesamt } \\
\text { Ordentliche Mitglieder in Österreich } \\
\text { Ordentliche Mitglieder im Ausland }\end{array}$ & \begin{tabular}{|r|}
402 \\
376 \\
26 \\
\end{tabular} & $\begin{array}{r}123 \\
123 \\
0 \\
\end{array}$ & $\begin{array}{r}525 \\
499 \\
26 \\
\end{array}$ & 33 & 266 & 299 & 435 & 389 & 824 \\
\hline Studierende, Schüler & 39 & 22 & 61 & 0 & 240 & 240 & 39 & 262 & 301 \\
\hline Familienmitglieder & 0 & 11 & 11 & 0 & 16 & 16 & 0 & 27 & 27 \\
\hline $\begin{array}{l}\text { Firmen, Institute, Schulen } \\
\text { beitragsfreie Mitglieder } \\
\text { Institute, Schulen } \\
\text { Firmenmitglieder } \\
\text { Förderer }\end{array}$ & $\begin{array}{r}32 \\
3 \\
16 \\
11 \\
2\end{array}$ & $\begin{array}{l}0 \\
0 \\
0 \\
0 \\
0\end{array}$ & $\begin{array}{r}32 \\
3 \\
16 \\
11 \\
2\end{array}$ & 0 & 3 & 3 & 32 & 3 & 35 \\
\hline Mitglieder gesamt & 500 & 156 & 656 & 35 & 525 & 560 & 535 & 681 & 1.216 \\
\hline
\end{tabular}

Tab. 4: Mitgliederstand der ÖGG Ende 2017 ( $\mathrm{mM}=$ mit MÖGG, oM = ohne MÖGG)

\begin{tabular}{|l|r|r|r|}
\hline & Wien & Innsbruck & $\begin{array}{r}\text { ÖGG } \\
\text { gesamt }\end{array}$ \\
\hline Mitglieder Anfang 2017 & $\mathbf{6 5 9}$ & $\mathbf{5 9 3}$ & $\mathbf{1 . 2 5 2}$ \\
Eintritte & 29 & 8 & 37 \\
Todesfälle & 5 & 2 & 7 \\
Austritte, Streichungen & 27 & 39 & 66 \\
\hline Mitglieder Ende 2017 & $\mathbf{6 5 6}$ & $\mathbf{5 6 0}$ & $\mathbf{1 . 2 1 6}$ \\
\hline Mitgliedergewinn/-verlust & -3 & -33 & -36 \\
\hline
\end{tabular}

Tab. 5: Mitgliederbewegung 2017 im Stammverein Wien und im Zweigverein Innsbruck 


\subsection{Anpassung der Mitgliedsbeiträge ab 2019}

(Helmut WoHLSCHLÄGL)

Auf Antrag des Vorstandes der ÖGG, den dieser in seiner Sitzung am 13. April 2018 einstimmig gefasst hatte, wurde in der Hauptversammlung - ebenfalls einstimmig - beschlossen, die Mitgliedsbeträge ab dem Jahr 2019 moderat zu erhöhen.

\begin{tabular}{|l|r|r|}
\hline \multirow{2}{*}{ Art der Mitgliedschaft } & \multicolumn{2}{c|}{ Mitgliedsbeitrag (EUR) } \\
\cline { 2 - 3 } & bis 2018 & ab 2019 \\
\hline Ordentliches Mitglied der ÖGG * & 42,00 & 48,00 \\
\hline Ordentliches Mitglied der ÖGG im Ausland * & 47,00 & 53,00 \\
\hline Studentisches Mitglied (gilt längstens bis zum 27. Lebensjahr) * & 28,00 & 32,00 \\
\hline Familienmitglied ** & 7,00 & 12,00 \\
\hline Mitglied einer Fachgruppe ** & 21,00 & 24,00 \\
\hline Studierende/r als Mitglied einer Fachgruppe ** & 7,00 & 12,00 \\
\hline Sponsoren und Förderer & \multicolumn{2}{|}{} \\
\hline Firmenmitgliedschaft, 5-facher Jahresbeitrag & 210,00 & 240,00 \\
\hline Förderer der ÖGG, 10-facher Jahresbeitrag & 420,00 & 480,00 \\
\hline Förderer der ÖGG, 40-facher Jahresbeitrag & $1.680,00$ & $1.920,00$ \\
\hline Lebenslanges Mitglied, 25-facher Jahresbeitrag & $1.050,00$ & $1.200,00$ \\
\hline
\end{tabular}

* Mit Bezug der Fachzeitschrift „Mitteilungen der Österreichischen Geographischen Gesellschaft“ (MÖGG) und der vierteljährlichen Informations-Zeitschrift „Geographie aktuell“

** Ohne Bezug der „Mitteilungen der Österreichischen Geographischen Gesellschaft“, nur mit Bezug von „Geographie aktuell““

Tab. 6: Überblick über die Anpassung der ÖGG-Mitgliedsbeiträge ab 2019 in allen Mitgliedskategorien gemäß einstimmigem Beschluss der Hauptversammlung

Bereits in der Hauptversammlung des Vorjahres am 19. April 2017 war auf die laufenden Kostensteigerungen (z. B. bei den Betriebs- und Mietkosten für die Geschäftsstelle und bei den Personalkosten) hingewiesen und eine maßvolle Anpassung der Mitgliedsbeiträge angedacht worden. Der Präsident betonte in der Hauptversammlung bei der Begründung des Antrags des Vorstands, dass die Mitgliedsbeiträge trotz der generellen Kostensteigerungen, die neben den Personalkosten und den Betriebs- und Mietkosten der Kanzlei in besonderem Maß auch bei den Postgebühren zu verzeichnen sind, seit 2009 (also seit zehn Jahren!) nicht mehr angepasst worden sind.

In diesem Zusammenhang merkte er auch an, dass die Mitgliedsbeiträge zahlreicher anderer Gesellschaften (bei vergleichbaren oder sogar geringeren Leistungen für die Mitglieder) deutlich höher sind (z. B. Gesellschaft für Erdkunde zu Berlin EUR 100,-; Verband der Geographen an Deutschen Hochschulen EUR 100,-; Deutscher Verband für Angewandte Geographie EUR 110,-), während bei der ÖGG der jährliche Mitgliedsbeitrag für das ordentliche Vollmitglied nur von EUR 42,- auf EUR 48,- erhöht werde.

Zur Begründung dieser Anpassung der Mitgliedsbeiträge ab 2019 wies der Präsident auch auf folgenden Vergleich hin:

- Steigerung des Verbraucherpreisindex von März 2010 bis März 2018: 15,7 Prozent innerhalb von acht Jahren; 
- Steigerung der Personalkosten der ÖGG von 2009 bis 2018: 22,4 Prozent innerhalb von neun Jahren;

- Erhöhung der Mitgliedsbeiträge von 2009 bis 2019: Ordentliches Vollmitglied von EUR 42,- auf EUR 48,- = nur 14,3 Prozent innerhalb von zehn Jahren.

- Die Erhöhung ist also niedriger als die Inflationsrate,

- sie ist weitaus niedriger als die Steigerung der Personalkosten der ÖGG

- und sie umfasst nur EUR 6,- pro Jahr, das sind 50 Cent pro Monat.

Alle anderen Mitgliedsbeiträge werden aliquot erhöht.

\subsection{Ergebnisse der Wahlen}

(Helmut WoHLSCHLÄGL)

\section{Wahl des Präsidenten / der Präsidentin für die Funktionsperiode 2018 bis 2021}

Die dreijährige Funktionsperiode (2015-2018) des aktuellen Präsidenten, Univ.-Prof. i.R. Mag. Dr. Helmut Wohlschlägl, läuft mit dieser Hauptversammlung aus. Helmut WoHLSCHLÄGL hat sich bereit erklärt, noch eine weitere Funktionsperiode (2018-2021) als Präsident der ÖGG zur Verfügung zu stehen, falls der Gesamtvorstand und die Hauptversammlung dies wünschen.

Einstimmiger Beschluss des Vorstands in seiner Sitzung vom 13. April 2018: Der Vorstand der ÖGG befürwortet die Wiederkandidatur des aktuellen Präsidenten für die Funktionsperiode 2018 bis 2021 und empfiehlt der Hauptversammlung die Wiederwahl von Helmut WoHLSCHLÄGL.

Weitere Kandidatinnen oder Kandidaten stellten sich seit der offiziellen Veröffentlichung der Wahl des Präsidenten/der Präsidentin in „Geographie aktuelle“, Nr. 34 (I/2017) nicht zur Verfügung.

\begin{tabular}{|l|r|}
\hline Wahl des Präsidenten(der Präsidentin) & \\
\hline Abgegebene Stimmen & 18 \\
\hline Univ.-Prof. i.R. Mag. Dr. Helmut WoHLSCHLÄGL & 17 \\
\hline Ao. Univ.-Prof. Mag. Dr. Martin HeINTEL & 1 \\
\hline
\end{tabular}

Tab. 7:Ergebnis der Wahl des Präsidenten/der Präsidentin der ÖGG

Der Präsident ist damit mit einem nahezu einstimmigen Ergebnis für die Funktionsperiode 2018 bis 2021 wiedergewählt. Die anwesenden Mitglieder begrüßen dieses Ergebnis sehr und gratulieren dem alten und neuen Präsidenten herzlich.

\section{Rücktritte von Vorstandsmitgliedern}

Vier Vorstandsmitglieder der ÖGG haben bei der Vorstandssitzung am 13. April 2018 oder brieflich mitgeteilt, dass sie aus dem Vorstand ausscheiden möchten. Es handelt sich um

- Univ.-Prof. Dipl.-Geogr. Dr. Thomas Glade (statt ihm als Vertreter des Instituts für Geographie und Regionalforschung der Universität Wien in der ÖGG: Patrick SAKDAPOLRAK);

- Mag. Helene KaUtz (Leitung der ÖGG-Bibliothek; diese Funktion wird vorerst nicht nachbesetzt);

- Priv.-Doz. Dr. Martin Mergili (statt ihm als neuer Leiter der Fachgruppe „Österreichische Forschungsgruppe für Geomorphologie und Umweltwandel“ - geomorph.at: Ronald PöPPL);

- Mag. Peter Alexander Rumpolt (zuständig für Ausstellungen; stellv. Redaktionsleiter von „Geographie aktuell“; diese Funktion wird vorerst nicht nachbesetzt). 
Die Hauptversammlung der ÖGG dankt den vier aus dem Vorstand ausscheidenden Mitgliedern per einstimmigem Beschluss sehr herzlich für ihre langjährige ehrenamtliche Tätigkeit im Interesse der ÖGG

\section{Wahl oder Wiederwahl von Vorstandsmitgliedern}

Der Vorstand hat in seiner Sitzung vom 13. April 2018 beschlossen, die folgenden ÖGG-Mitglieder zur Wiederwahl (da ihre gemäß Satzung der ÖGG dreijährige Funktionsperiode abgelaufen ist) oder zur Neuwahl in den Vorstand vorzuschlagen. Die Wahlen ergaben bei den in der Hauptversammlung anwesenden Mitgliedern bei jeweils 18 abgegebenen Stimmen folgende Zustimmungswerte für die Wieder- oder Neuwahl:

\begin{tabular}{|l|c|}
\hline Wiederwahl & \\
\hline Univ.-Prof. Dr. Martin CoY & 18 \\
\hline Univ.-Prof. Dr. Heike EGNER & 18 \\
\hline Prof. Dr. Gerhard FASCHING & 13 \\
\hline Mag. Norbert HACKNER-JAKLIN & 17 \\
\hline Ao. Univ.-Prof. i.R. Dr. Albert HofMAYER & 16 \\
\hline Univ.-Doz. Dr. Wolfgang SCHWARZ & 17 \\
\hline Univ.-Doz. Dr. Christian STAUDACHER & 16 \\
\hline Neuwahl & 18 \\
\hline $\begin{array}{l}\text { Mag. Dr. Ronald PöPPL, BA (als neuer Leiter der Fachgruppe „Österreichische } \\
\text { Forschungsgruppe für Geomorphologie und Umweltwandel““-geomorph.at) }\end{array}$ & 18 \\
\hline $\begin{array}{l}\text { Univ.-Prof. Dipl.-Geogr. Dr. Patrick SAKDAPOLRAK (als neuer Vertreter des In- } \\
\text { stituts für Geographie und Regionalforschung in der ÖGG) }\end{array}$ & 18 \\
\hline
\end{tabular}

Tab. 8: Ergebnis der Wieder- bzw. Neuwahl in den Vorstand der ÖGG

\section{Wahl von Rechnungsprüfern}

Für die Funktion von Rechnungsprüfern standen für das Vereinsjahr 2018 neuerlich zur Verfügung: Mag. Andreas Palkovics und Dr. Klaus Wilhelmer. Beide wurden einstimmig in diese Funktion wiedergewählt. Die ÖGG dankt für ihre wertvolle Arbeit als Rechnungsprüfer!

\section{Geographie-Fonds der ÖGG}

\subsection{Verleihung der wissenschaftlichen Preise für $\mathbf{2 0 1 6}$}

Die wissenschaftlichen Preise des Geographie-Fonds der ÖGG für das Jahr 2016 wurden in Band 158/2015 der MÖGG sowie ab Jänner 2016 auf der Homepage der ÖGG (http://www.geoaustria.ac.at) ausgeschrieben. Bis zum festgesetzten Einreichtermin sind insgesamt 15 Einreichungen eingegangen: für den Hans-Bobek-Preis zwei Dissertationen, für den Förderungspreis der ÖGG acht Diplom- oder Masterarbeiten und für den Leopold-Scheidl-Preis für Wirtschaftsgeographie eine Dissertation und vier Masterarbeiten. 
Entsprechend den Statuten des Geographie-Fonds mussten im Begutachtungsverfahren von Albert HofmaYer, dem Geschäftsführer des Fonds, insgesamt 28 Gutachterinnen und Gutachter, davon 22 aus dem Ausland, eingeworben werden; diese haben ihre Gutachten sehr gewissenhaft erstellt, wofür Ihnen im Namen der ÖGG herzlich zu danken ist. Die intensive Arbeit der elfköpfigen Jury über die Preisvergabe 2016 ergab in der Jurysitzung vom 25. Oktober 2017 folgende Entscheidung:

- Hans-Bobek-Preis 2016: Alle zwei eingereichten Arbeiten hatten ein hohes Niveau. Einige Jurymitglieder waren jedoch dennoch der Meinung, dass die Arbeiten den sehr hohen wissenschaftlichen Anforderungen, die an Dissertationen zu stellen sind, denen der Hans-Bobek-Preis verliehen werden soll, nicht völlig genügen würden. Nach ausführlicher Diskussion ergab dann das geheime Abstimmungsverfahren unter den Jury-Mitgliedern, dass keine der beiden eingereichten Arbeiten die gemäß den Statuten des Geographie-Fonds erforderliche Zwei-Drittel-Mehrheit erreichte. Der Hans-Bobek-Preis 2016 wurde daher nicht vergeben.

- Förderungspreis der ÖGG 2016: In der Sitzung der Jury wurde vor allem die Qualität des Großteils der insgesamt acht eingereichten Arbeiten besonders betont, so dass es der Jury nicht leicht fiel, eine Entscheidung zu treffen. Nach ausführlicher Diskussion ergab dann der erste Wahlgang des geheimen Abstimmungsverfahrens kein eindeutiges Ergebnis und der zweite Wahlgang einen Stimmengleichstand zwischen zwei besonders herausragenden Arbeiten. Die Jury beschloss daher, den Förderungspreis 2016 ausnahmsweise doppelt zu vergeben. und zwar für die von Herrn Johannes HerburGer MA am Institut für Geographie und Regionalforschung der Universität Wien verfasste Masterarbeit zum Thema „Die sozialräumliche Struktur der LEADER-Region Vorderland - Walgau - Bludenz. Auf dem Weg zum alpinen Postsuburbia? “ und für die von Herrn Clemens HILlER MSc am Fachbereich Geographie und Geologie der Universität Salzburg verfasste Masterarbeit zum Thema ,,Temperaturvariabilitäten an der Basis der winterlichen Schneedecke. Raumzeitliche Veränderungsmuster am Maurerkogel (Kitzsteinhorn, Hohe Tauern) ".

- Leopold-Scheidl-Preis für Wirtschaftsgeographie 2016: Auch bei diesem Preis erwiesen sich mehrere der insgesamt fünf eingereichten Arbeiten nach Auffassung der internationalen Gutachter und der Jury als preiswürdig. Nach ausführlicher Diskussion verblieben dann zunächst zwei Masterarbeiten in der engeren Wahl, wobei das geheime Abstimmungsverfahren im zweiten Durchgang dann schließlich eine klare Mehrheit für die am Institut für Geographie der Universität Bremen verfasste Masterarbeit von Herrn Felix WiLmSEn MA zum Thema „Je mehr, desto weniger? Das Verhältnis von Wachstumskritik und alternativen Wirtschaftskonzepten des Postwachstums zum kapitalistischen System " erbrachte.

Die Vergabe der drei wissenschaftlichen Preise für 2016 (zweimal Förderungspreis der ÖGG; Leopold-Scheidl-Preis für Wirtschaftsgeographie) erfolgte in feierlicher Form im Rahmen der Jahresschlussveranstaltung (Weihnachtsfeier) der ÖGG am Dienstag, 5. Dezember 2017. Die ÖGG gratuliert den drei Preisträgern sehr herzlich. Kurzberichte über die drei preisgekrönten Arbeiten wurden in „Geographie aktuell“, Nr. 35, 2018, Seite 8 (HerbURGER, Förderungspreis), Nr. 36, 2018, Seite 4 (Hiller, Förderungspreis) und Nr. 35, 2018, Seite 9 (WILMSEN, Scheidl-Preis) abgedruckt.

\subsection{Ausschreibung der Preise des Geographie-Fonds der ÖGG für 2019}

\section{Hans-Bobek-Preis 2019}

Zum Gedenken an den Ehrenpräsidenten der Österreichischen Geographischen Gesellschaft (ÖGG) sowie in Würdigung der großen Verdienste von Universitätsprofessor Dr. Dr. h.c. Hans 
Bовек als einem der bedeutendsten und international hoch angesehenen Geographen wird seit dem Jahr 1991 jährlich ein Förderungspreis in der Höhe von EUR 2.000,- ausgeschrieben, der von Frau Dr. Maria BoBeK-FeSL gestiftet wird.

Eingereicht werden können in deutscher oder englischer Sprache verfasste Dissertationen, Habilitationsschriften oder andere gleichwertige, von einer Person selbstständig verfasste wissenschaftliche Arbeiten, die in den Jahren 2018 oder 2019 fertiggestellt und approbiert worden sind. Zugelassen sind Personen im Alter bis zu 45 Jahren.

Ausgeschlossen sind Personen, die zur Zeit der Bewerbung um den Hans-Bobek-Preis als Vorstandsmitglieder, Rechnungsprüfer oder Angestellte der ÖGG wirken, ferner solche, bei denen das Verfahren der Approbation der Dissertation oder das Habilitationsverfahren noch nicht rechtskräftig abgeschlossen ist.

Bei den eingereichten Arbeiten muss es sich um thematisch und methodisch besonders herausragende wissenschaftliche Leistungen handeln, die geeignet erscheinen, die geographische Wissenschaft wesentlich zu bereichern und voranzutreiben. Die dazu notwendige Feststellung trifft eine vom Vorstand der Österreichischen Geographischen Gesellschaft eingesetzte Jury des Geographie-Fonds der ÖGG. Der Preis wird unter Ausschluss des Rechtsweges zuerkannt.

Sollte keine der eingereichten Arbeiten den zu fordernden Standard erreichen, bleibt es der Österreichischen Geographischen Gesellschaft vorbehalten, von einer Preisverleihung Abstand zu nehmen und den Geldbetrag des Hans-Bobek-Preises auf Folgejahre zu übertragen. Die Einreichung muss spätestens bis 31. Dezember 2019 bei der Geschäftsstelle der ÖGG, A-1070 Wien, Karl-Schweighofer-Gasse 3/7 (E-Mail: oegg.geographie@univie.ac.at), mit einem gedruckten Exemplar und einer elektronischen Version samt Bewerbungsschreiben, Lebenslauf und nach Möglichkeit mit bisher vorliegenden Gutachten erfolgen.

Die Jury des Geographie-Fonds der Österreichischen Geographischen Gesellschaft trifft ihre Entscheidung in ihrer Sitzung im Frühherbst des Jahres 2020 unter Einbeziehung von jeweils zwei Fachgutachten pro eingereichter Arbeit. Die eingereichten Unterlagen verbleiben bei der ÖGG.

Helmut WOHLSCHLÄGL

Präsident

\section{Förderungspreis der ÖGG 2019}

Die Österreichische Geographische Gesellschaft (ÖGG) vergibt seit 1995 alljährlich einen Förderungspreis in der Höhe von EUR 1.000,-. Die Mittel stammen zu 75 Prozent aus den Erträgen des Geographie-Fonds der ÖGG und zu 25 Prozent aus einem Beitrag des Zweigvereins Innsbruck der ÖGG.

Dieser Preis wird für thematisch und methodisch hervorragende Diplom- und Masterarbeiten aus allen Teilgebieten der Geographie verliehen, die von einer Person selbstständig verfasst und in den Jahren 2018 oder 2019 fertiggestellt und approbiert worden sind. Es können sich nur Personen bewerben, die zum Zeitpunkt der Bewerbung das 35. Lebensjahr noch nicht vollendet haben und deren eingereichte Arbeit an einer österreichischen Universität approbiert wurde. Ausgeschlossen von der Bewerbung sind Vorstandsmitglieder, Rechnungsprüfer oder Angestellte der ÖGG.

Die eingereichten Arbeiten müssen spätestens bis 31. Dezember 2019 in der Geschäftsstelle der ÖGG eingetroffen sein. Erwartet werden: ein gebundenes Exemplar und eine elektronische Version der Arbeit mit Bewerbungsschreiben, Lebenslauf und bisher vorliegenden Gutachten, zu senden an die Österreichische Geographische Gesellschaft, A-1070 Wien, Karl-Schweighofer-Gasse 3/7 (E-Mail: oegg.geographie@univie.ac.at).

Die Jury des Geographie-Fonds der Österreichischen Geographischen Gesellschaft trifft ihre Entscheidung in ihrer Sitzung im Frühherbst des Jahres 2020 unter Einbeziehung von jeweils zwei 
Fachgutachten pro eingereichter Arbeit. Der Preis wird unter Ausschluss des Rechtsweges zuerkannt. Die eingereichten Unterlagen verbleiben bei der ÖGG.

Helmut WoHLSCHLÄGL

Präsident

\section{Leopold-Scheidl-Preis für Wirtschaftsgeographie 2019}

Die Österreichische Geographische Gesellschaft (ÖGG) vergibt für hervorragende Leistungen auf dem Gebiet der Wirtschaftsgeographie den Leopold-Scheidl-Preis 2019 in Höhe von EUR 1.000,-.

Dieser Preis wird für thematisch und methodisch hervorragende Dissertationen, Diplom- und Masterarbeiten aus Wirtschaftsgeographie und verwandten Fachgebieten verliehen, die von einer Person selbstständig verfasst und in den Jahren 2018 oder 2019 fertiggestellt und approbiert worden sind. Es besteht keine Einschränkung nach Alter oder Wohnsitz der einreichenden Person. Ausgeschlossen von der Bewerbung sind Vorstandsmitglieder, Rechnungsprüfer oder Angestellte der ÖGG.

Die eingereichten Arbeiten müssen spätestens bis 31. Dezember 2019 in der Geschäftsstelle der ÖGG eingetroffen sein. Erwartet werden: ein gebundenes Exemplar und eine elektronische Version der Arbeit mit Bewerbungsschreiben, Lebenslauf und bisher vorliegenden Gutachten, zu senden an die Österreichische Geographische Gesellschaft, A-1070 Wien, Karl-Schweighofer-Gasse 3/7 (E-Mail: oegg.geographie@univie.ac.at).

Die Jury des Geographie-Fonds der Österreichischen Geographischen Gesellschaft trifft ihre Entscheidung in ihrer Sitzung im Frühherbst des Jahres 2020 unter Einbeziehung von jeweils zwei Fachgutachten pro eingereichter Arbeit. Der Preis wird unter Ausschluss des Rechtsweges zuerkannt. Die eingereichten Unterlagen verbleiben bei der ÖGG.

Helmut WOHLSCHLÄGL

Präsident

\section{Herfried-Berger-Preis 2019}

1) In Klagenfurt a.W. besteht der Fonds zur Förderung wissenschaftlicher Arbeiten auf dem Gebiet der Geographie in memoriam Prof. Herfried Berger.

2) Das Kuratorium dieses Fonds verleiht 2019 einen Preis für herausragende Diplomarbeiten oder Dissertationen aus dem Gebiet der Geographie.

3) Schwerpunkte der Arbeiten sollen dem Gebiet der Ostalpen oder jenem des östlichen Afrika, den Forschungsgebieten von Herfried Berger, zuordenbar sein.

4) Die eingereichten Arbeiten sollen aktuell und zu einer Preisverleihung noch nicht eingereicht worden sein.

5) Über die Preisverleihung entscheidet das Kuratorium des Fonds unter Einbeziehung von Fachgutachten unter Ausschluss des Rechtsweges.

6) Die zu begutachtenden Arbeiten mögen möglichst umgehend an Prof. h.c. Univ.-Doz. Dr. Peter JoRDAN, Österreichische Akademie der Wissenschaften, Institut für Stadt- und Regionalforschung (A-1010 Wien, Postgasse 7/4/2) oder an Univ.-Prof. Dr. Friedrich Zimmermann, Karl-Franzens-Universität Graz (A-8010 Graz, Heinrichstraße 36) mit formlosem Begleitschreiben zugesandt werden; Kennwort: Herfried-Berger-Preis.

Für das Kuratorium:

Peter Jordan, Friedrich Zimmermann, Kuratoriumsmitglieder 